\title{
Mitochondrial ROS production and subsequent ERK phosphorylation are necessary for temperature preconditioning of isolated ventricular myocytes
}

\author{
Y Bhagatte ${ }^{1}$, D Lodwick ${ }^{2}$ and N Storey ${ }^{*, 1}$
}

Hypothermia and hypothermic preconditioning are known to be profoundly cardioprotective, but the molecular mechanisms of this protection have not been fully explained. In this study, temperature preconditioning $\left(16^{\circ} \mathrm{C}\right)$ was found to be cardioprotective in isolated adult rat ventricular myocytes, enhancing contractile recovery and preventing calcium dysregulation after oxidative stress. Hypothermic preconditioning preserved mitochondrial function by delaying the pathological opening of the mitochondrial permeability transition pore (mPTP), whereas transient mPTP flickering remained unaltered. For the first time, reactive oxygen species (ROS) from the mitochondria are shown to be released exclusively during the hypothermic episodes of the temperature-preconditioning protocol. Using a mitochondrially targeted ROS biosensor, ROS release was shown during the brief bursts to $16{ }^{\circ} \mathrm{C}$ of temperature preconditioning. The ROS scavenger N-(2-mercaptopropionyl) glycine attenuated ROS accumulation during temperature preconditioning, abolishing the protective delay in MPTP opening. Temperature preconditioning induces ROS-dependant phosphorylation of the prosurvival kinase extracellular signal-regulated kinase (ERK)1/2. ERK1/2 activation was shown to be downstream of ROS release, as the presence of a ROS scavenger during temperature preconditioning completely blocked ERK1/2 activation. The cardioprotective effects of temperature preconditioning on MPTP opening were completely lost by inhibiting ERK1/2 activation. Thus, mitochondrial ROS release and ERK1/2 activation are both necessary to signal the cardioprotective effects of temperature preconditioning in cardiac myocytes.

Cell Death and Disease (2012) 3, e345; doi:10.1038/cddis.2012.84; published online 5 July 2012

Subject Category: Internal Medicine

Mitochondria are the gatekeepers of cardiac myocyte death or survival, particularly in ischaemic heart disease. The opening of the mitochondrial permeability transition pore (mPTP) is considered to be the 'point of no return', after which the myocyte is irreversibly committed to necrotic or apoptotic death pathways. ${ }^{1}$ This is responsible for the production of necrotic regions that are routinely observed after long periods of ischaemia followed by reperfusion. ${ }^{2}$ When the mPTP opens, low molecular weight molecules $(<1.5 \mathrm{kDa})$ equilibrate across the inner membrane, resulting in mitochondrial swelling and outer membrane rupture. Oxidative phosphorylation is uncoupled when the mitochondrial membrane potential depolarises so that ATP can no longer be synthesised leading to ATP depletion and necrotic cell death. ${ }^{1}$ The situation is exacerbated as mitochondria begin to consume ATP through reversal of the F1/F0 ATPase in an effort to maintain the mitochondrial membrane potential. ${ }^{3}$ Strong evidence suggests that inhibiting the $\mathrm{MPTP}$ is a powerful means to protect the heart against ischaemia/ reperfusion injury. ${ }^{4,5}$ One example of an $\mathrm{mPTP}$ inhibitor is cyclosporine $A(C s A)$, which binds to cyclophilin-D, a core component of the mPTP. CsA has been shown to significantly decrease infarct size in both an animal model and in humans, ${ }^{6,7}$ and to delay the time to MPTP opening in single cell analysis. ${ }^{8}$ In addition to irreversible MPTP opening, the MPTP has been reported to flicker, ${ }^{9}$ which may serve as a way for mitochondria to remove excess mitochondrial reactive oxygen species (ROS) and calcium during respiration. ${ }^{10}$

Cardioprotection is a phenomenon whereby the myocardium can be protected from damage by ischaemia/reperfusion injury. Cardioprotection can be conferred by many protocols, including pre-treatment with mitochondrial uncouplers, such as DNP, and hormones, such as insulin, and profound protection is observed with ischaemic preconditioning, in which short bursts of mild ischaemia are applied before prolonged ischaemia. ${ }^{11-13}$ More recently, profound cardioprotective effects of temperature preconditioning have been described, which involve brief bursts of hypothermia before ischaemia. $^{14}$

Hypothermia is now considered to be one of the most potent cardioprotective strategies available and is used clinically to protect the heart against ischaemia in a number of surgical

\footnotetext{
${ }^{1}$ Department of Cell Physiology and Pharmacology, College of Medicine, Biological Sciences and Psychology, University of Leicester, Leicester, UK and ${ }^{2}$ Department of Cardiovascular Sciences, University of Leicester, Leicester, UK

${ }^{*}$ Corresponding author: N Storey, Department of Cell Physiology and Pharmacology, College of Medicine, Biological Sciences and Psychology, University of Leicester, Maurice Shock Medical Sciences Building, University Road, P.O. Box 138, Leicester, Leicestershire LE1 9HN, UK. Tel: + 44 (0)116 2297145 ; Fax: + 44 (0)116 252 5045; E-mail: ns140@leicester.ac.uk

Keywords: hypothermia; cardioprotection; ERK; ischaemia/reperfusion; cardiac; preconditioning

Abbreviations: mPTP, mitochondrial permeability transition pore; ROS, reactive oxygen species; MPG, N-(2-mercaptopropionyl) glycine; ERK, extracellular signalregulated kinases; RISK, reperfusion injury salvage kinases; MI, metabolic inhibition solution; TMRM, tetramethylrhodamine, methyl ester; CsA, cyclosporine A Received 26.3.12; revised 4.5.12; accepted 14.5.12; Edited by A Finazzi-Agró
} 
procedures, such as coronary bypass, valve repair and transplantation. ${ }^{15,16}$ Many animal models have shown that hypothermia is protective, including a study in dogs in which whole-body hypothermia protected the ischaemic myocardium. ${ }^{17}$ Also, hypothermia localised to the myocardial risk zone early after the onset of coronary occlusion salvaged $18 \%$ of the risk region and significantly decreased the area of infarct in rabbit studies. ${ }^{15}$ Since then, myocardial hypothermia by endovascular cooling has been shown to decrease infarct size in pigs if administered during ischaemia, but not to be beneficial if administered at reperfusion. ${ }^{18}$ It is proposed that a $1-{ }^{\circ} \mathrm{C}$ drop in body temperature slows metabolic rate by approximately $8 \%,{ }^{19}$ which may preserve ATP levels and explain some of the protection to the ischaemic myocardium by hypothermia. However, modest hypothermia $\left(34^{\circ} \mathrm{C}\right)$ only has a very mild effect on ATP levels, yet is still very protective, which suggests that the protection is more complex than just a case of energy preservation alone. ${ }^{20}$ The molecular mechanisms of hypothermic preconditioning are not fully understood.

The signalling pathways that are activated in response to a cardioprotective stimuli are not fully elucidated, but what is known is that preventing pathological MPTP opening underlies the infarct-limiting effects and may represent the end point of cardioprotective signalling pathways. ${ }^{8,21}$ One set of signalling molecules characterised as having a role in myocyte survival signalling is the reperfusion injury salvage kinases (RISK). ${ }^{22}$ Involvement of the pro-survival kinase, extracellular signal-regulated kinase (ERK)1/2, a member of the RISK family, in preconditioning has been shown in a number of cardioprotective strategies, including ischaemic preconditioning. ${ }^{21,23}$ Indeed, ERK1/2 phosphorylation is maintained during a $30 \mathrm{~min}$ ischemic period in isolated rabbit hearts perfused with hypothermic solutions, whereas ERK1/2 phosphorylation falls markedly after 10 min during normothermic ischaemia. ${ }^{24}$ ROS, possibly released from the mitochondria by MPTP flickering during ischemic preconditioning, has been postulated as a signal that may initiate subsequent survival pathways. ${ }^{21}$

In this study, the focus was on the effect of temperature preconditioning using rapid bursts of profound $\left(16^{\circ} \mathrm{C}\right)$ hypothermia in an isolated rat ventricular myocyte model of ischaemia/reperfusion injury. The effects of temperature preconditioning on contractile recovery and calcium homeostasis following exposure to simulated ischaemia/reperfusion injury were investigated. Using two independent fluorescence assays, the effect of temperature preconditioning on pathological mPTP opening was tested. How temperature preconditioning affected MPTP flickering rates was assessed with a calcein-cobalt quench assay. Using a novel mitochondrially targeted ROS biosensor, ${ }^{25}$ the timing of ROS release from the mitochondria during the temperature preconditioning protocol was investigated, and how this affects the downstream ERK1/2 activation is shown.

\section{Results}

Temperature preconditioning improves recovery of contractile function and calcium homeostasis following simulated ischaemia/reperfusion injury. Temperature preconditioning, like ischaemic preconditioning, has been reported in whole-heart studies to significantly increase cardioprotection. ${ }^{14}$ To investigate the molecular mechanisms of temperature preconditioning in cardioprotection, adult rat hearts were dissociated into single ventricular myocytes and subjected to simulated ischaemia/reperfusion injury. The myocytes were perfused with Tyrode buffer and contracted in response to electrical field stimulation (1 Hz; Figure 1ai). Ischaemia was simulated by rapidly preventing myocyte ATP production using a 'metabolic inhibition (MI) solution'. After several minutes, the myocytes lost their ability to contract and shortened into a state of rigor contracture (Figure 1aii). After $7 \mathrm{~min}$ of using the $\mathrm{Ml}$ solution, reperfusion was simulated by re-energising the myocytes by perfusion with Tyrode buffer to wash out the inhibitors and reintroduce metabolic substrates for ATP generation. The number of myocytes that had regained their contractile function was quantified after $10 \mathrm{~min}$ of reperfusion (Figure 1aiii).

Isolated ventricular myocytes, temperature preconditioned by two 2-min incubations at $16^{\circ} \mathrm{C}$, showed a significant increase in recovery of contractile function when compared with control cells (51.6 \pm 5.7 versus $28.1 \pm 3.4 \%$, Figure $1 \mathrm{~b})$. This improvement in contractile recovery was comparable to those produced by other known cardioprotective agents: the mitochondrial uncoupler, dinitrophenol (DNP) and the mPTP inhibitor, CsA (56.5 \pm 8.5 and $58.8 \pm 6.8 \%$, respectively; Figure 1b).

The effects of preconditioning protocols on calcium homeostasis after simulated ischaemia/reperfusion of contracting myocytes were investigated using Fura2-loaded myocytes to report intracellular calcium changes. Temperature preconditioning had no effect on calcium homeostasis before MI (Figures 2ai and bi). However, following cessation of field stimulation at the end of the $\mathrm{Ml} /$ reperfusion protocol, intracellular calcium was significantly lower for temperature preconditioned cells $(132 \pm 17 \mathrm{nM})$ compared with control (301 $\pm 52 \mathrm{nM}$; Figures 2aii and b). Temperature preconditioning resulted in a significant increase in the percentage of myocytes with a cellular calcium estimate $<150 \mathrm{nM}$ (Figure 2bii). Temperature preconditioning resulted in a significant improvement in the percentage of myocytes that regained synchronous calcium transients after $\mathrm{Ml} /$ reperfusion compared with control. (Figure 2biii). Also, both pretreatment with the mitochondrial uncoupler DNP and the presence of CsA protected against the loss of calcium homeostasis after $\mathrm{Ml} /$ reperfusion (Figures $2 \mathrm{c}$ and $\mathrm{d}$ ).

Temperature preconditioning delays pathological MPTP opening in a model of reperfusion injury. The MPTP is considered to be the central determinant of myocyte fate following ischaemia/reperfusion injury, and cardioprotective signalling cascades are thought to converge on the MPTP to prevent opening and thus inhibit myocyte death. ${ }^{7,26-28}$ The effect of temperature preconditioning on time to mPTP opening was investigated with two independent assays to quantify mitochondrial membrane potential in intact cardiac myocytes. In the first assay, the myocytes were loaded with a relatively high tetramethylrhodamine, methyl ester (TMRM) concentration $(5 \mu \mathrm{M})$, which accumulates in the mitochondria and autoquenches to become non-fluorescent. Depolarisation of the mitochondrial membrane potential, due to MPTP 

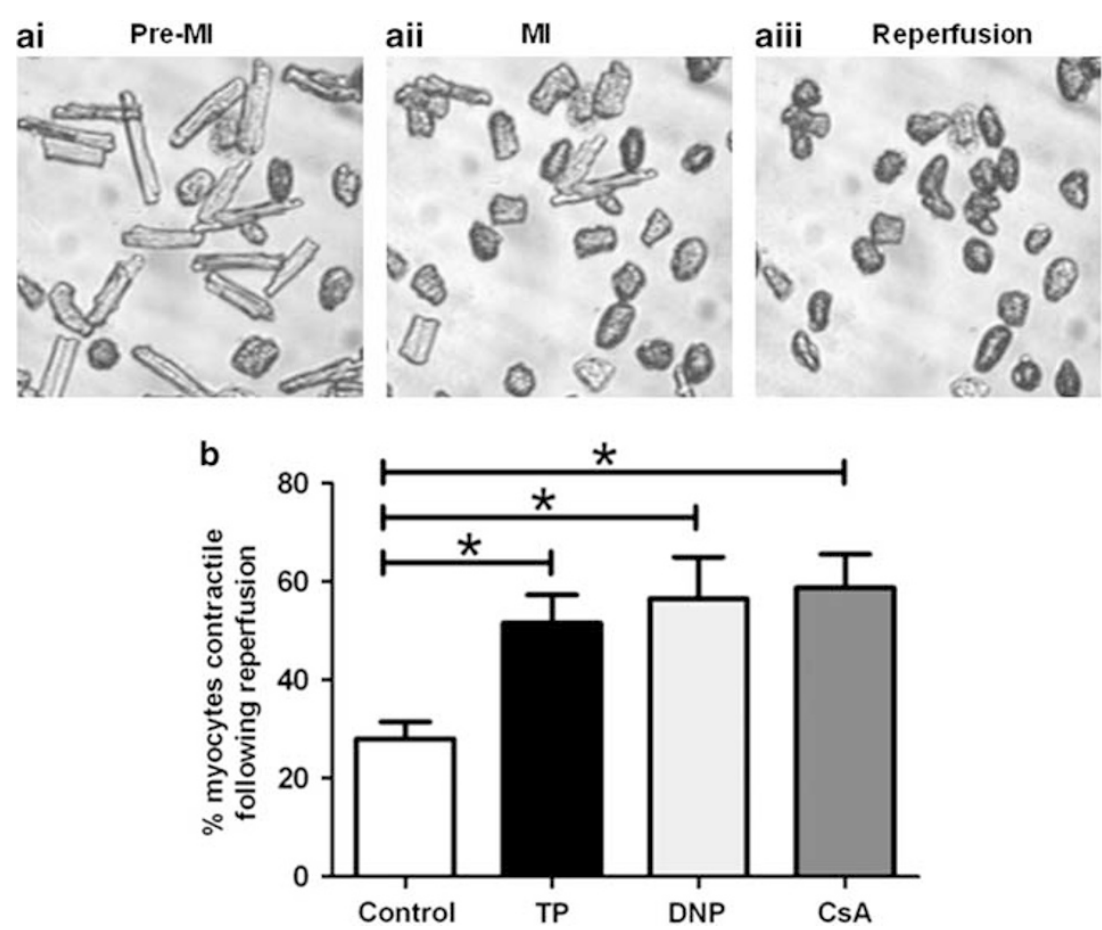

Figure 1 The effects of temperature preconditioning on the ability of the myocytes to regain contractile function after metabolic inhibition (MI) and reperfusion. (ai) Representative image of isolated rod-shaped cardiac myocytes before Ml; (aii) following $7 \mathrm{~min}$ of $\mathrm{Ml}$, cardiac myocytes fail to respond to electrical field stimulation and undergo rigor contracture; (aiii) upon reperfusion, myocytes hypercontract and their ability to regain contractile function was assessed after 10 min. (b) Mean data of the percentage of myocytes that recovered contractile function following $7 \mathrm{~min}$ of $\mathrm{Ml}$ and $10 \mathrm{~min}$ of reperfusion for control (without hypothermia or drug pre-treatment), $28.1 \pm 3.4$, $n=199,3$; temperature preconditioning (TP), $51.6 \pm 5.7, n=225,3$; 2,4-dinitrophenol (DNP $50 \mu \mathrm{M}$ ) preconditioning, $56.5 \pm 8.5, n=230,3$; and cyclosporine A (CsA, $4 \mu \mathrm{M}$ ) treatment, $58.8 \pm 6.8, n=187$, 3. Data are expressed as mean \pm S.E.M., $n=$ number of cells, number of hearts. ${ }^{\star} P<0.05$

opening, causes TMRM to be redistributed into the cytosol and dequenched, giving an increase in fluorescence. The mPTP opening was induced by oxidative stress caused by continuous illumination of TMRM at $546 \mathrm{~nm} .{ }^{8}$ Under these conditions, both temperature preconditioning and DNP resulted in a significant delay in the time to fluorescence increase (mPTP opening) relative to control (2.5- and 1.7-fold increase, respectively) (Figures $3 a-c)$. The presence of the known MPTP inhibitor, CsA, also resulted in a significant delay in the time required for MPTP opening to occur (Figure 3c). To exclude the possibility of a non-specific effect of CsA on calcineurin accounting for the delay in MPTP opening, experiments were conducted in the presence of $\mathrm{FK}$ 506, a calcineurin inhibitor, and no delay in MPTP opening was observed (Figure 3c). Conversely, carboxyatractyloside, an MPTP opener, resulted in a significant acceleration in the time to MPTP opening (Figure 3c).

As TMRM was used to both record and stimulate MPTP opening in this assay, a second assay was developed to confirm the results. Continuous illumination was avoided, and the myocytes were loaded with a relatively low concentration of TMRM $(50 \mathrm{nM})$, which accumulates in the mitochondria, but not at a concentration high enough to quench so the fluorescence remains high. Myocyte MPTP opening was induced by ROS generated by addition of xanthine and xanthine oxidase. Upon mPTP opening, the fluorescence signal decreased as the TMRM redistributed into the cytosol and then into the bathing solution. No photodamage-induced
mPTP opening was observed using this protocol, as fluorescence was measured at a sampling rate of $0.2 \mathrm{~Hz}$ (Figures 4ai and ii). The ROS donor, xanthine and xanthine oxidase caused a significant decrease in myocyte fluorescence (Figures $4 \mathrm{a}$ and $\mathrm{b}$ ). Temperature preconditioning resulted in a significant delay in mitochondrial mPTP opening relative to control $(15.1 \pm 1.3 \mathrm{~min}$ compared with $10.3 \pm 0.9 \mathrm{~min}$, respectively) (Figures $4 \mathrm{~b}$ and $\mathrm{c}$ ). The presence of the MPTP inhibitor, CsA, also resulted in a significant delay in the time to MPTP opening compared with control $(15.5 \pm 1.1 \mathrm{~min}$ compared with $10.3 \pm 0.9 \mathrm{~min}$, respectively, Figure 4c).

Transient MPTP flickering is not altered by temperature preconditioning. It has been demonstrated that non-pathological, transient mPTP flickering occurs under basal conditions, and may serve as a way to regulate mitochondrial $\mathrm{ROS}$ and $\mathrm{Ca}^{2+}$ concentration within myocytes. ${ }^{29}$ The effect of temperature preconditioning on non-pathological mPTP flickering was investigated using a calcein-cobalt quench assay. Isolated ventricular myocytes were co-loaded with calcein-AM $(1 \mu \mathrm{M})$ and cobalt chloride $(1 \mathrm{mM})$. Calcein-AM readily crosses cell and mitochondrial membranes, whereas cobalt chloride is spatially restricted to the cytosol. Cobalt chloride quenches cytosolic calcein fluorescence to reveal a punctate pattern of mitochondrial calcein fluorescence. During basal mPTP flickering, calcein is released from mitochondria into the cytosol where its fluorescence is 

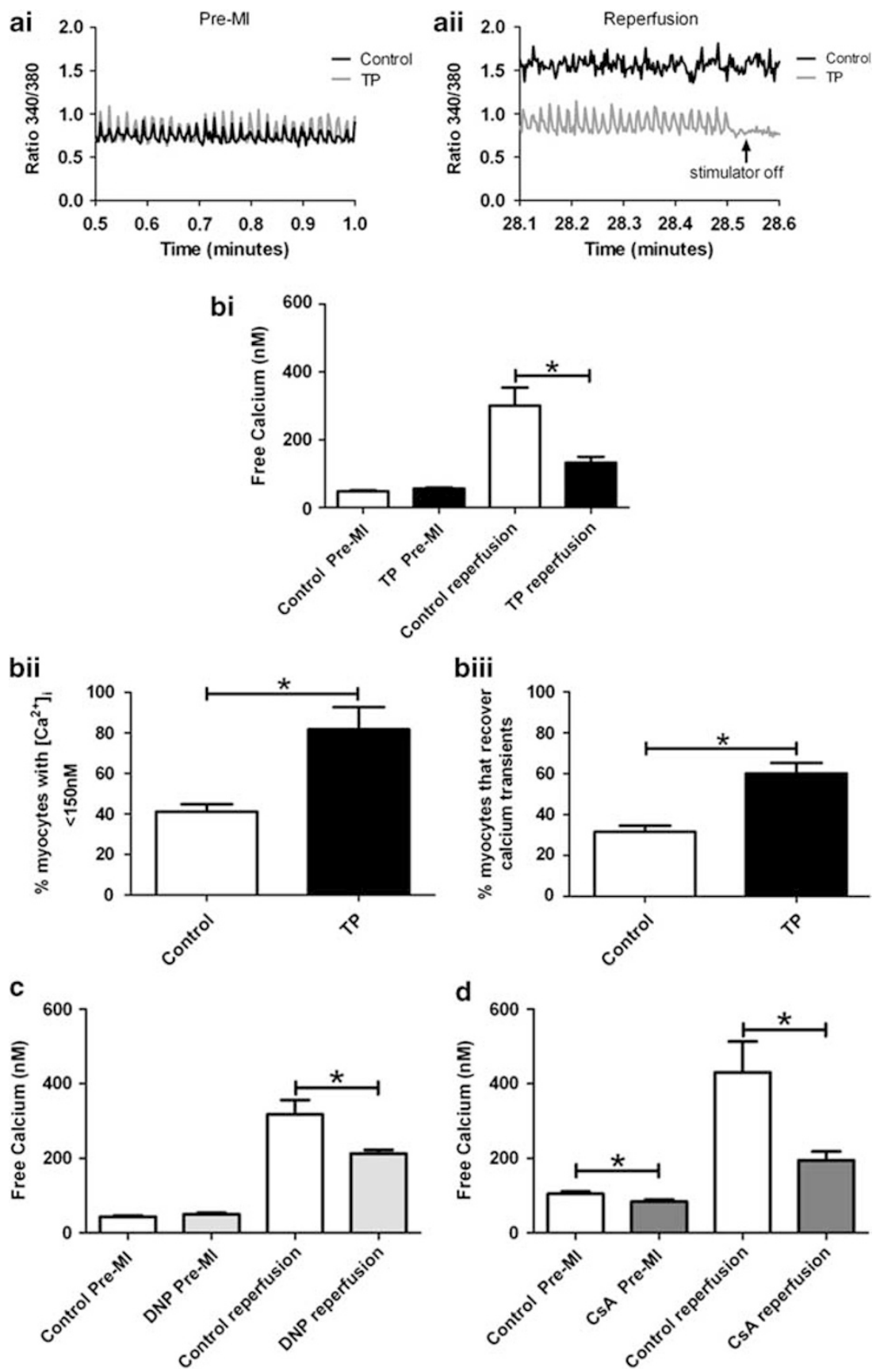

Figure 2 The effects of temperature preconditioning on calcium homeostasis of ventricular myocytes after metabolic inhibition (MI) and reperfusion. (ai) Representative 340/380 ratio from representative control and temperature-preconditioned (TP), Fura-2AM-loaded myocytes contracting in response to electrical field stimulation (1 Hz), and (aii) following $7 \mathrm{~min}$ of $\mathrm{Ml}$ and $10 \mathrm{~min}$ of reperfusion. The stimulator was turned off following $10 \mathrm{~min}$ of reperfusion (indicated by the arrow) for all measurements of basal-free $\mathrm{Ca}^{2+}$ concentration. (bi) Estimated free $\mathrm{Ca}^{2+}$ concentrations (nM) before and after Ml reperfusion on same day matched control myocytes for control $(301.3 \pm 52.7$, $n=69,3)$ compared with temperature-preconditioned myocytes $(132.1 \pm 17.6, n=67,3)$; (bii) the percentage of control (without hypothermia pre-treatment; $41.10 \pm 3.677, n=69,3)$ and temperature preconditioned $(81.71 \pm 11.00, n=67,3)$ that had $\left[\mathrm{Ca}^{2+}\right]_{\mathrm{i}}<150 \mathrm{nM}$ after Ml reperfusion; and (biii) the percentage of control $(31.56 \pm 3.020, n=69,3)$ and temperature-preconditioned myocytes $(60.14 \pm 5.095, n=67,3)$ that recovered synchronous calcium transients after Ml-reperfusion. Estimated free $\mathrm{Ca}^{2+}$ concentrations $(\mathrm{nM})$ before and after Ml reperfusion on same day matched control myocytes for (c) control $(317.8 \pm 38.5 \mathrm{nM}, n=44,3)$ compared with DNP preconditioned $(212.9 \pm 10.0 \mathrm{nM}, n=42$, 3); (d) control $(430.9 \pm 82.2, n=70,3)$ compared with CsA treatment $(194.5 \pm 23.5, n=88,3)$. Data are expressed as mean \pm S.E.M., $n=$ number of cells, number of hearts, ${ }^{*} P<0.005$

quenched by cobalt chloride. This is observed as a change in the fluorescence pattern from a mitochondrial punctuate pattern (Figure 5ai) to a decreased and diffuse fluorescence (Figure 5aii). Temperature-preconditioned myocytes showed a decrease in cellular fluorescence over the 30 -min period to $78.8 \pm 4.1 \%$ of initial fluorescence, which was similar to that observed in control myocytes $(80.2 \pm 7.2 \%$ of initial fluorescence; Figures $5 b$ and $c)$. However, when experiments were 


\section{ai}

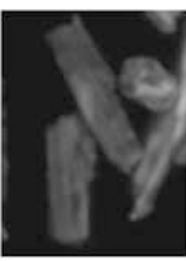

$$
\text { b }
$$

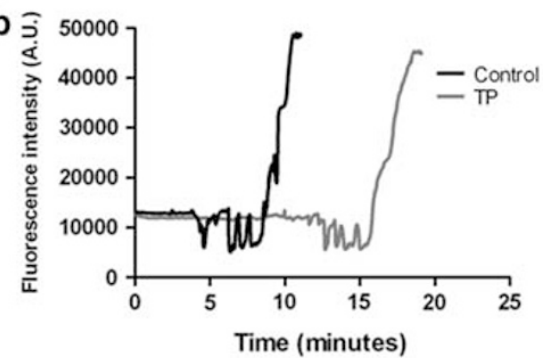

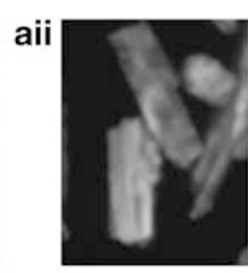

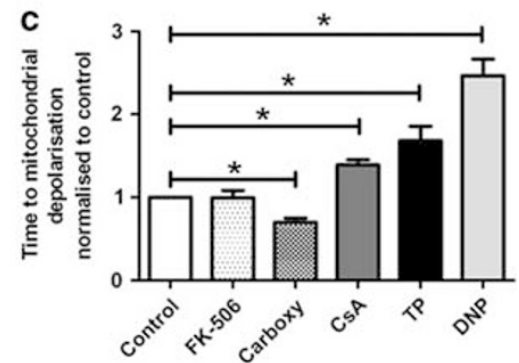

Figure 3 The effect of temperature preconditioning on the time to photodamage-induced mPTP opening in isolated myocytes. Representative image of TMRM ( $5 \mu \mathrm{M})$ loaded myocytes at (ai) time $=0 \mathrm{~min}$, before photodamage. The myocytes show a low level of fluorescence, as the TMRM fluorescent signal is autoquenched within the mitochondria; and (aii) time $=5.29 \mathrm{~min}$, following a period of continuous illumination, the mPTP opens, which is indicated by the global increase in cellular fluorescence. (b) Representative traces showing the changes in TMRM fluorescence during the course of continuous illumination for control (black) and temperature-preconditioned myocytes (grey). (c) The time to irreversible TMRM fluorescence increase was measured in control myocytes $(n=353,3)$ and was compared with the time taken in myocytes that were treated with FK-506 $(1 \mu \mathrm{M}), n=44$; carboxyatractyloside, an mPTP opener, (carboxy, $7.5 \mu \mathrm{M}), n=51,3$; cyclosporine, an mPTP blocker (CsA, $4 \mu \mathrm{M}), n=162,3$; temperature preconditioning (TP), $n=126,3$; and dinitrophenol (DNP, $50 \mu \mathrm{M}$ ), $n=90,3, n=$ number of cells, number of hearts. ${ }^{*} P<0.005$
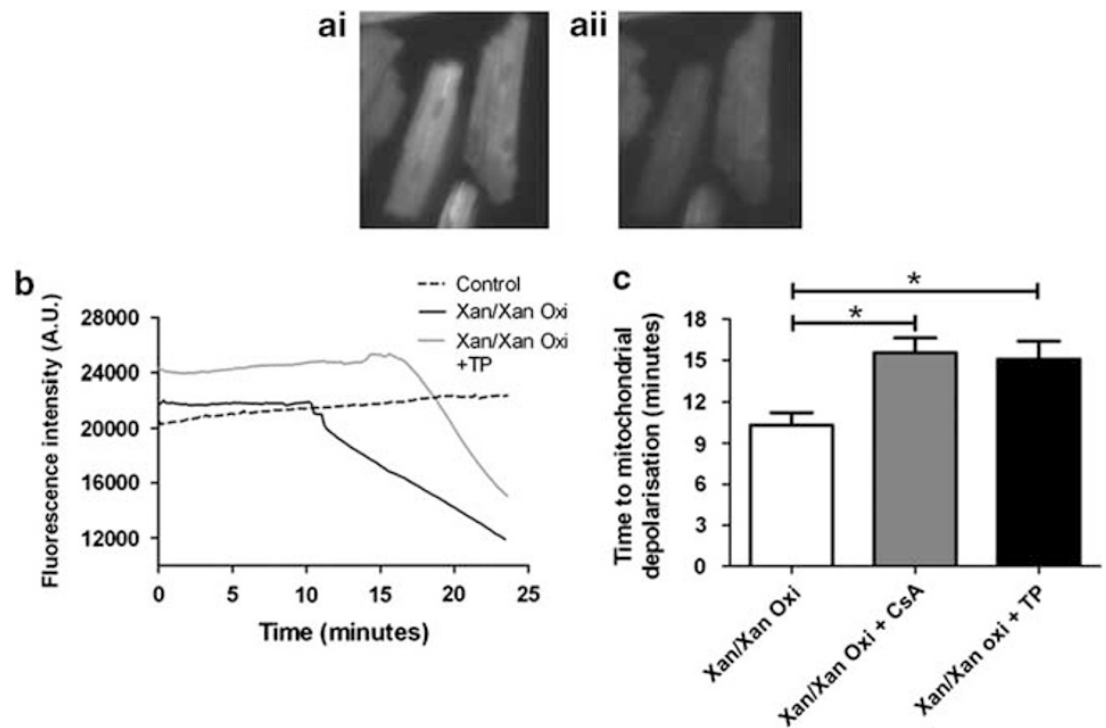

Figure 4 The effect of temperature preconditioning on ROS-induced mPTP opening. (ai) TMRM (50 nM)-loaded control myocytes displaying relatively high, mitochondrially localised fluorescence at time $=0 \mathrm{~min}$. (aii) Myocytes were superfused with xanthine $(2 \mathrm{mM})$ and xanthine oxidase $(20 \mathrm{mU})$ to induce $\mathrm{mPTP}$ opening, and fluorescence was measured at time $=15 \mathrm{~min}$. (b) Representative traces from control and temperature-preconditioned myocytes challenged with xanthine and xanthine oxidase, and control myocytes unchallenged with xanthine and xanthine oxidase. (c) Mean time to fluorescence decrease for control myocytes, $10.3 \pm 0.9$ min, $n=17,3$; cyclosporine-A-treated myocytes (CsA, $4 \mu \mathrm{M}$ ), $15.5 \pm 1.1 \mathrm{~min}, n=14,3$; and temperature-preconditioned (TP) myocytes, $15.1 \pm 1.3 \mathrm{~min}, n=18,3$. Data are expressed as mean \pm S.E.M., $n=$ number of cells, number of hearts, ${ }^{\star} P<0.05$

conducted in the presence of the known MPTP inhibitor, CsA, there was no decrease in cellular fluorescence $(101.2 \pm 2.9 \%$ of initial fluorescence; Figures $5 b$ and $c)$.

Mitochondrial ROS production is essential for the protective effects of temperature preconditioning. Although excessive amounts of ROS are detrimental to myocyte viability, small amounts have been shown to have beneficial effects and are crucial to certain cardioprotective interventions, including ischaemic preconditioning. ${ }^{21,30}$ To investigate the involvement of ROS in the signalling cascade of temperature preconditioning, isolated ventricular myocytes were infected with adenovirus, containing DNA encoding a mitochondrially targeted superoxide biosensor. ${ }^{25}$ After a 48-h infection, the myocytes expressing the biosensor were characterised by low-level fluorescence that increased after the addition of exogenous ROS. To confirm the sensitivity of the biosensor to ROS, myocytes were challenged with 

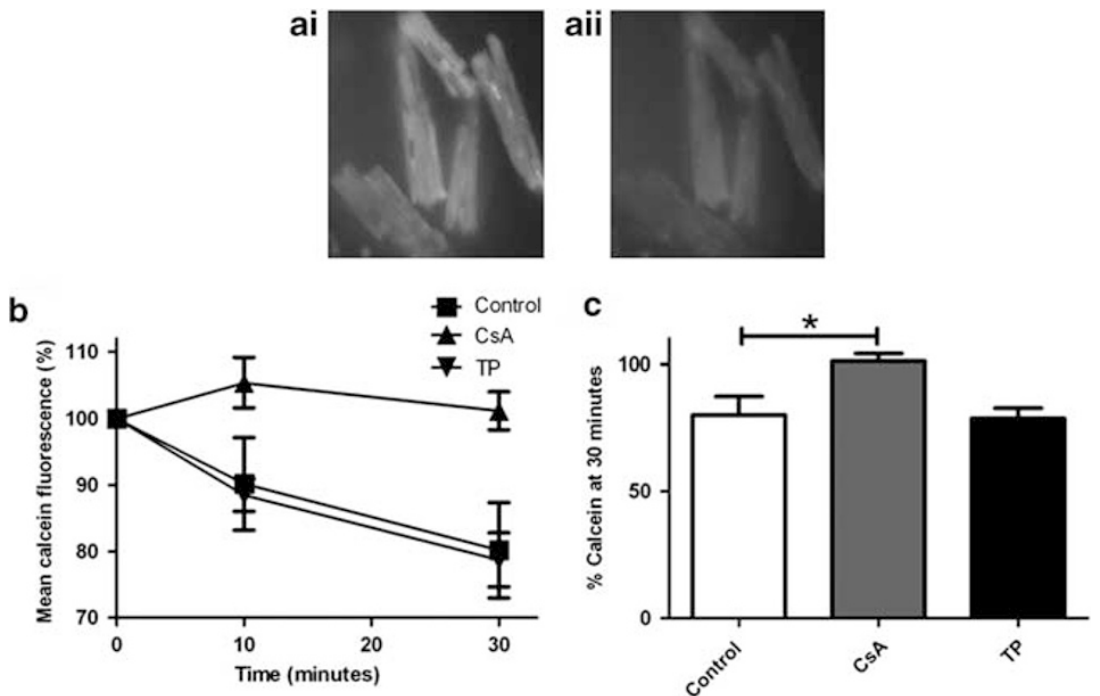

Figure 5 The effect of temperature preconditioning on mPTP flickering. The calcein-cobalt quench assay was used to access the basal rate of mPTP flickering. (ai) Control myocytes loaded with calcein and cobalt at time $=0 \mathrm{~min}$ and (aii) time $=30 \mathrm{~min}$. (b) Calcein fluorescence was measured at times 0,10 and $30 \mathrm{~min}$ expressed as a percentage of initial fluorescence (time $=0 \mathrm{~min}$ ). (c) Fluorescence values of myocytes were compared between control, $80.20 \pm 7.19 \%, n=13,3$; cyclosporine A (CsA, $4 \mu \mathrm{M}), 101.2 \pm 2.871 \%, n=11,3$; and temperature-preconditioned (TP) $78.75 \pm 4.070 \%, n=12,3$ at $30 \mathrm{~min}$. Data are expressed as mean \pm S.E.M., $n=$ number of cells, number of hearts, ${ }^{*} P<0.05$

exogenous ROS by addition of xanthine and xanthine oxidase, and an increase in fluorescence was detected (Figures $6 \mathrm{a}$ and $\mathrm{b}$ ). Infected myocytes were loaded with mitoTracker deep red, and co-localisation confirmed the mitochondrial localisation (Figure 6c). The table (Figure 6d) shows the infection efficiency for four representative experiments. The mitochondrial ROS increased, indicated by an increase in biosensor fluorescence, during the 2-min decrease in temperature to $16^{\circ} \mathrm{C}$ (Figure 6e). Fluorescence rose rapidly upon the switch to the lower temperature and quickly reversed when the temperature was restored to $37^{\circ} \mathrm{C}$. To confirm the increase in ROS during the hypothermic steps of the temperature-preconditioning protocol, the experiments were repeated in the presence of a ROS scavenger, N-(2-mercaptopropionyl) glycine (MPG). Scavenging ROS significantly decreased the $16^{\circ} \mathrm{C}$-induced increase in fluorescence observed during the temperaturepreconditioning protocol (Figure 6e and f). Significantly, MPG also prevented the protective delay in time to mitochondrial depolarisation in response to photodamage, which was observed with temperature preconditioning (Figure 6g).

ERK activation is downstream of mitochondrial ROS production and is necessary for the protection induced by temperature preconditioning. The RISK pathway is a protective pathway shown to be activated by a number of cardioprotective interventions, including ischaemic preconditioning. ${ }^{31}$ A number of kinases have been demonstrated to be involved in the RISK pathway, one of which is the ERK.

The activation of ERK by temperature preconditioning was investigated by western blotting for active phosphorylated ERK (pERK). Control myocytes and myocytes that were analysed $10 \mathrm{~min}$ after the completion of the temperature preconditioning protocol were examined for pERK levels, with total ERK included as a loading control (Figure 7a and b). Temperature-preconditioned myocytes were found to have significantly greater levels of pERK compared with untreated control myocytes. The temperature-preconditioning-induced increase in pERK was abolished if temperature preconditioning was conducted in the presence of ERK activation inhibitors, either PD98059 or U0126 (Figure 7a and b). Furthermore, the temperature-preconditioning-induced activation of ERK was also abolished by the presence of the ROS scavenger, MPG, during the temperature-preconditioning protocol (Figure 7a and b).

The functional importance of the temperature-preconditioning-induced ERK activation was investigated by the temperature-preconditioning of myocytes in the presence of an ERK inhibitor, and testing the delay to photodamage-induced mPTP opening. Inhibition of ERK with either PD98095 or U0126 abolished the protective delay in time to mitochondrial membrane depolarisation conferred by temperature preconditioning (Figure 7c).

\section{Discussion}

The results of this study demonstrate that temperature preconditioning by two brief episodes of profound hypothermia $\left(16^{\circ} \mathrm{C}\right)$ results in strong cardioprotection of isolated ventricular myocytes against oxidative stress. Temperature preconditioning delays pathological mPTP opening, which is considered to be the end effector for preconditioning pathways. However, non-pathological transient mPTP opening or 'flickering' remains unaltered after the bursts of hypothermia. Mitochondrial ROS were produced during the brief bursts of hypothermia, which was shown to be necessary to signal cardioprotection. ERK activation is known to have an essential role in cardioprotective signalling, and mitochondrial 


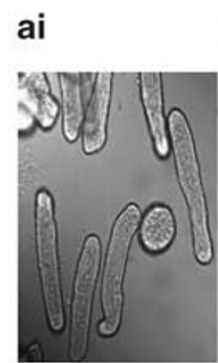

aii

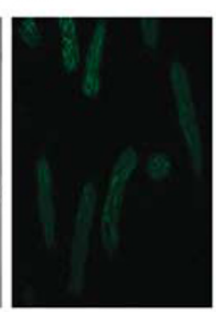

aiii

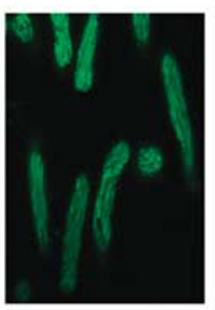

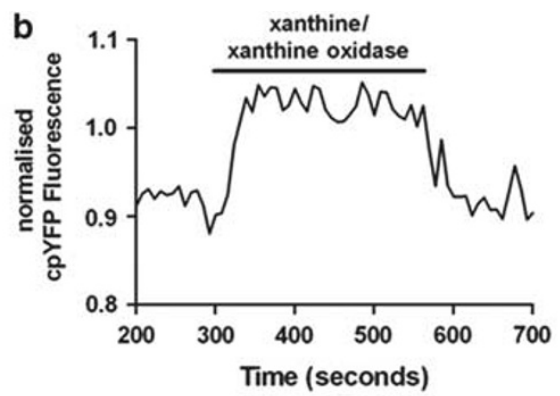

ci

cii

ciii

civ

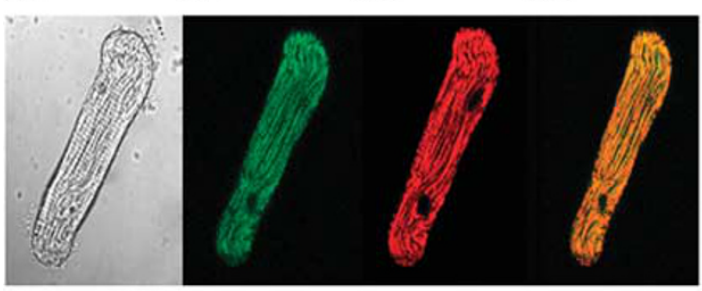

d

\begin{tabular}{|c|c|c|c|}
\hline $\begin{array}{c}\text { superoxide } \\
\text { biosensorinfection }\end{array}$ & $\begin{array}{c}\text { fields of view } \\
\text { counted }\end{array}$ & $\begin{array}{c}\text { total } \\
\text { cells }\end{array}$ & $\begin{array}{c}\text { \% infection } \\
\text { efficiency }\end{array}$ \\
\hline 1 & 2 & 8 & 88 \\
\hline 2 & 6 & 23 & 61 \\
\hline 3 & 5 & 12 & 50 \\
\hline 4 & 4 & 15 & 66 \\
\hline Total/Average & 17 & 58 & 66 \\
\hline
\end{tabular}
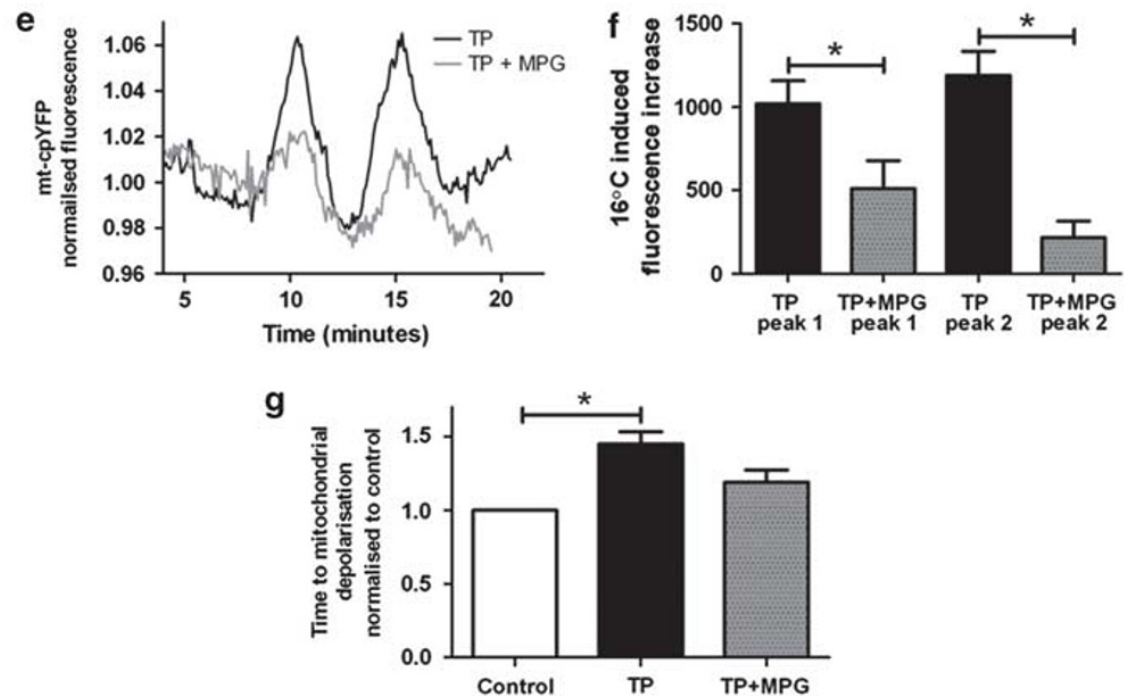

Figure 6 Temperature preconditioning increases mitochondrial ROS production. (ai) A bright-field image of a representative field of view of adenovirally infected mt-cpYFP-expressing myocytes. (aii) A fluorescent image of the same myocytes at the beginning of the experiment. (aiii) Shows the same myocytes after exposure to xanthine $(2 \mathrm{mM})$ and xanthine oxidase $(20 \mathrm{mU})$. (b) Representative trace showing the effect of xanthine and xanthine oxidase on the fluorescence of an mt-cpYFP-expressing myocyte. (c) Confocal images of an mt-cpYFP-expressing (48 h) myocyte also loaded with mitoTracker deep red. (ci) Bright-field image. (cii) ROS biosensor fluorescence when excited at $488 \mathrm{~nm}$ and challenged with xanthine and xanthine oxidase. (ciii) MitoTracker deep red fluorescence when excited at $640 \mathrm{~nm}$, and (civ) merged image of the fluorescence from the ROS biosensor and mitoTracker deep red. The orange indicates the co-localisation of the ROS biosensor and the mitochondria. (d) Table showing the infection efficiency of the ROS biosensor adenovirus. Infected myocytes were counted from a selection of fields of view from four separate infections and the average percentage of infected myocytes is shown. (e) Representative traces of mt-cpYFP-expressing control and ROS scavenger, MPG (300 $\mu \mathrm{M})$, treated myocytes during the temperaturepreconditioning protocol. (f) The mean peak fluorescence (arbitrary units) observed during the temperature preconditioning protocol for control (peak $1=1020 \pm 136.5$, peak $2=1190 \pm 143.9, n=19,3$ ) and MPG-treated (peak $1=510.4 \pm 167.3$, peak $2=217.2 \pm 98.58, n=18,3$ ) myocytes, ${ }^{*} P<0.05$. (g) The fold increase in time to mPTP opening measured by loading myocytes with TMRM $(5 \mu \mathrm{M})$ and continuously illuminating for control, $n=41,3$, temperature preconditioned, $n=42,3$, or MPG (300 $\mu \mathrm{M})$ treated temperature-preconditioned myocytes, $n=45$, 3. Data are expressed as mean \pm S.E.M., $n=$ number of cells, number of hearts, ${ }^{*} P<0.0001$

ROS production was shown in this study to be required for ERK1/2 phosphorylation in temperature preconditioning.

The cardioprotective effects of hypothermia have been known for some time, but the mechanism of protection has not been clear. Although certainly preservation of ATP content during cooling is an attractive hypothesis, the profound protection observed, especially in mild hypothermia $\left(32{ }^{\circ} \mathrm{C}\right)$, cannot be explained fully by ATP preservation alone. ${ }^{20}$
Hypothermic conditioning has been used advantageously in many clinical intracardiac settings, especially in transplantation. ${ }^{18}$ Indeed, the use of therapeutic hypothermia has the potential to reduce myocardial infarct size. ${ }^{32}$ The signalling events, which initiate the hypothermic cardioprotection, are described here.

Two cycles of hypothermia to $16^{\circ} \mathrm{C}$ for $2 \mathrm{~min}$, interspersed with normothermia at $37^{\circ} \mathrm{C}$ for 3 min resulted in a significant 

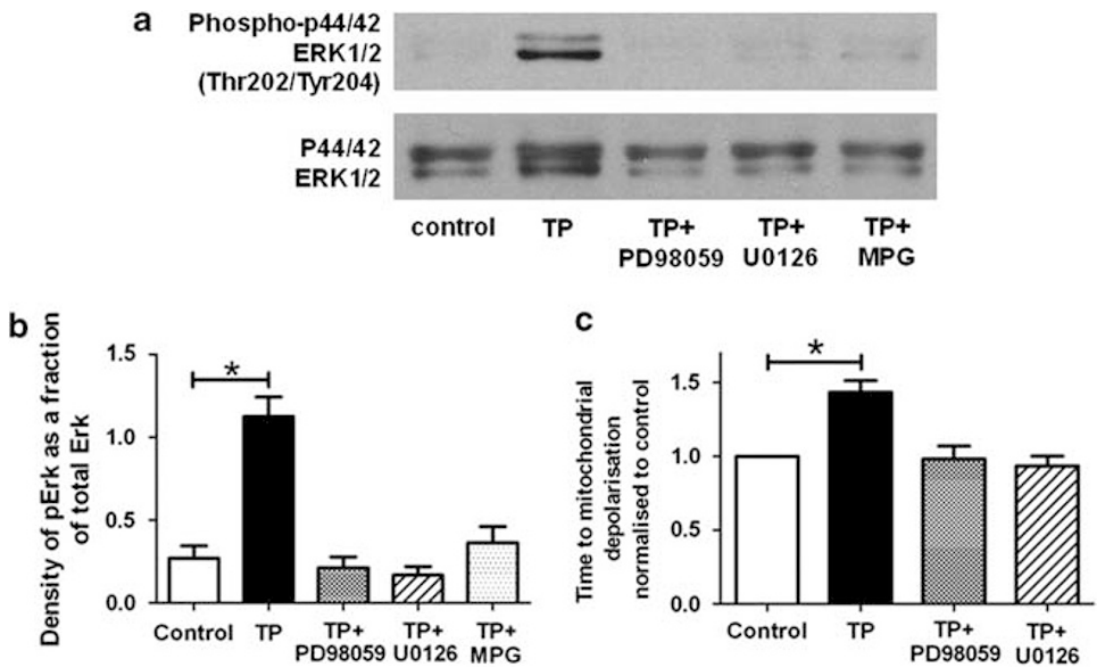

Figure 7 Temperature preconditioning increases ERK phosphorylation. (a) Representative western blot showing total ERK and ERK phosphorylation in response to temperature preconditioning with and without ERK inhibitors, PD98059 $(10 \mu \mathrm{M})$ or U0126 $(0.5 \mu \mathrm{M})$ and ROS scavenger MPG $(300 \mu \mathrm{M})$. (b) Average densitometry showing the effects of temperature preconditioning, and temperature preconditioning in the presence of ERK inhibitors and the ROS scavenger, MPG, on ERK phosphorylation, $n=3$. Western blots from three hearts, ${ }^{*} P<0.005$. (c) TMRM $(5 \mu \mathrm{M})$-loaded myocytes were continuously illuminated, and the time to mitochondrial depolarisation was measured. The fold increase relative to control $(n=38,3)$ in time to mPTP opening in response to temperature preconditioning $(n=47,3)$, and temperature preconditioning with ERK inhibitors, PD98059 $(10 \mu \mathrm{M}, n=18,3)$ or U0126 $(0.5 \mu \mathrm{M}, n=31,3) ; n=$ number of cells, number of hearts, ${ }^{*} P<0.005$

improvement in recovery of contractile function after simulated ischaemia/reperfusion injury. This shows that the signalling pathways involved in temperature preconditioning exist at the level of individual myocytes and are independent of any signalling influence from other cell types present within the heart, such as endothelial cells or fibroblasts. These results are consistent with the observation that temperature preconditioning in the isolated whole heart improves haemodynamic recovery, decreases arrhythmia and reduces necrotic damage when assessed during reperfusion. ${ }^{14}$ Previous reports investigating the response of ex vivo whole hearts found that preconditioning at $26^{\circ} \mathrm{C}$ gives optimal cardioprotection..$^{33}$ In this study, although $26^{\circ} \mathrm{C}$ preconditioning did result in cardioprotection, $16^{\circ} \mathrm{C}$ gave a larger and more consistent cardioprotective effect. The reasons for this discrepancy are unclear and may relate to the fact that the myocytes in this study are isolated.

Elevated calcium in myocytes following ischaemia/reperfusion injury has pathophysiological implications associated with cellular injury. High intracellular calcium underlies hypercontracture, development of areas of cell death by necrosis, ${ }^{34,35}$ and the activation of pro-apoptotic proteins and calcium-dependent proteases. ${ }^{36}$ Temperature preconditioning led to a significant increase in the number of myocytes with healthy calcium levels (calcium $<150 \mathrm{nM}$ ) at the end of reenergisation compared with control myocytes. Temperature preconditioning also increased the percentage of myocytes that regained synchronous calcium transients and the proportion of myocytes that regained their contractile function in response to field stimulation. In addition, high mitochondrial matrix calcium concentration is a critical factor for the opening of the MPTP, which is believed to be central to myocyte death in the setting of ischaemia/reperfusion injury. ${ }^{37}$ Therefore, maintenance of calcium homeostasis of the hypothermically preconditioned myocytes may underlie many of the therapeutic benefits of temperature preconditioning already reported, including decreased infarct size. ${ }^{15}$

The opening of the MPTP was first proposed to be a mediator of cell death in the setting of acute ischaemia/ reperfusion injury by Crompton et al. in $1986 .{ }^{38}$ Since then, the opening of MPTP has become well established as the key determinant of cell fate, although how MPTP opening is modulated is not fully understood. ${ }^{39}$ Inhibiting MPTP opening with agents, such as CsA, result in cardioprotection. ${ }^{6,8}$ Temperature preconditioning of isolated cardiac myocytes delays the time to mitochondrial membrane potential depolarisation, which occurs on MPTP opening. This is consistent with reports that de-energised, isolated mitochondria harvested from temperature-preconditioned myocytes can inhibit calcium-induced mitochondrial swelling, which is also a reflection of the MPTP opening. ${ }^{14}$

Basal transient mPTP 'flickering' is proposed to allow release of excess mitochondrial ROS and calcium from the mitochondrial matrix. ${ }^{10}$ Moreover, hypoxic preconditioning has been shown to augment mPTP flickering, which was necessary for the development of cardioprotection. ${ }^{30}$ However, in this study, no changes in MPTP flickering were observed between the control and the temperature-preconditioned myocytes, but in all myocytes, the MPTP flickering was sensitive to CsA. This suggests that the modulation of MPTP that delays opening may be separate from that which affects basal flickering rates.

This study shows that mitochondrial ROS are produced during the hypothermic steps of temperature preconditioning, and that this is responsible for downstream activation of ERK1/2. A mitochondrially targeted superoxide biosensor ${ }^{25}$ was used to report mitochondrial ROS production in cardiac myocytes. Bursts of mitochondrial ROS production were detected exclusively during the two hypothermic $\left(16^{\circ} \mathrm{C}\right)$ periods of the temperature-preconditioning protocol. 
Hypothermia-induced mitochondrial ROS production is an obligatory signalling event required for temperature preconditioning, because the presence of a ROS scavenger during temperature preconditioning ablates the protective delay in mitochondrial depolarisation. ROS production during hypothermia may be due to a respiratory 'slow down'. ROS production was observed during metabolic depression of isolated mouse brain mitochondria that were maintained in hypothermic conditions. ${ }^{40}$ This may result from decreased activity of the F1/F0 ATPase and reduced oxygen consumption by the electron transport chain, which results in increased mitochondrial oxygen levels that can promote ROS production. Also consistent with these findings, hypothermia $\left(17^{\circ} \mathrm{C}\right)$ by cold perfusion of the isolated whole heart resulted in dihydroethidium fluorescence changes across the intact myocardial wall, suggesting an increase in ROS production. ${ }^{41}$

The activation of the pro-survival kinase, ERK1/2, is well established in cardioprotective pathways. ${ }^{21,23}$ The cardioprotective effects of ischaemic preconditioning are, in part, due to activation of ERK1/2 within the first few minutes of reperfusion. ${ }^{22}$ However, it has recently been shown that when isolated whole hearts were maintained in mild hypothermia $\left(35^{\circ} \mathrm{C}\right)$ during ischaemia, ERK $1 / 2$ phosphorylation was preserved for the duration of the ischaemic period instead of steadily declining that occurs in normothermia. ${ }^{24}$ The preservation of ERK $1 / 2$ during the hypothermic ischaemic period conferred a significant cardioprotection. ${ }^{24}$ Temperature preconditioning results in a robust phosphorylation of ERK1/2 when measured $10 \mathrm{~min}$ after the temperature-preconditioning stimulus, but before the ischaemic insult. The cardioprotection by temperature preconditioning was blocked by inhibition of ERK $1 / 2$ activation, showing that this is a key step in the cardioprotective pathway. A critical role of ROS in the activation of $E R K 1 / 2$ is shown, as scavenging ROS during the temperature preconditioning protocol was sufficient to abolish ERK $1 / 2$ activation and any subsequent cardioprotection. This shows that mitochondrial ROS production is upstream of and necessary for ERK1/2 activation in the temperaturepreconditioning signalling cascade in cardiac myocytes. Temperature preconditioning may therefore share common cardioprotective signalling pathways with many preconditioning stimuli, such as ischaemic preconditioning, and mitochondrial uncouplers, such as DNP. The common pathway may stem from a decrease in mitochondrial respiration, leading to increased release of ROS and subsequent ERK1/2 activation.

\section{Materials and Methods}

Isolation of adult rat ventricular myocytes. Adult male Wistar rats (200-350 g) were killed by cervical dislocation in accordance with the UK Animals (Scientific Procedures) Act, 1986. The heart was rapidly excised and placed in nominally $\mathrm{Ca}^{2+}$-free Tyrode solution titrated to $\mathrm{pH} 7.4$ with $\mathrm{NaOH}$, containing $135 \mathrm{mM} \mathrm{NaCl}, 6 \mathrm{mM} \mathrm{KCl}, 0.33 \mathrm{mM} \mathrm{NaH}_{2} \mathrm{PO}_{4}, 5 \mathrm{mMNa}$ pyruvate, $10 \mathrm{mM}$ glucose, $1 \mathrm{mM} \mathrm{MgCl}_{2}$ and $10 \mathrm{mM}$ HEPES. Within $1 \mathrm{~min}$, the heart was cannulated at the aorta and retrogradely perfused using a Langendorff apparatus for $6 \mathrm{~min}$ at a constant flow rate of $10 \mathrm{ml} / \mathrm{min}$ with $\mathrm{Ca}^{2+}$-free Tyrode at $37^{\circ} \mathrm{C}$, vigorously bubbled with $95 \% \mathrm{O}_{2} / 5 \% \mathrm{CO}_{2}$. The heart was then perfused with $\mathrm{Ca}^{2+}$-free Tyrode solution containing Type I collagenase (approximately 0.4 units $/ \mathrm{ml}$ ), protease type XIV (approximately 2.7 units $/ \mathrm{ml}$ ) and $0.05 \%$ bovine serum albumin for 7-10 min, to digest the heart. This was followed by perfusion for $3 \mathrm{~min}$ with Tyrode solution (containing $2 \mathrm{mM} \mathrm{CaCl}$ ) to remove the enzymes. Isolated ventricular myocytes were obtained by cutting the ventricular mass in two and gently agitating in Tyrode solution at $37^{\circ} \mathrm{C}$, in a shaking water bath. The isolation procedure typically yielded $70-90 \%$ viable rod-shaped myocytes. Following isolation, myocytes were stored in Tyrode solution at room temperature (approximately $21^{\circ} \mathrm{C}$ ) at a density of approximately 0.4 million cells $/ \mathrm{ml}$ and were used for experimentation within $10 \mathrm{~h}$. All compounds used throughout this study were acquired from Sigma-Aldrich (Gillingham, UK), unless otherwise stated.

$\mathrm{MI}$ and re-energisation. In this study, the $\mathrm{Ml}$ solution was the substrate-free Tyrode solution containing $140 \mathrm{mM} \mathrm{NaCl}, 6 \mathrm{mM} \mathrm{KCl}, 0.33 \mathrm{mM} \mathrm{NaH}_{2} \mathrm{PO}_{4}, 10 \mathrm{mM}$ sucrose, $1 \mathrm{mM} \mathrm{MgCl}_{2}, 2 \mathrm{mM} \mathrm{CaCl}$ and $10 \mathrm{mM} \mathrm{HEPES}$, and was titrated to $\mathrm{pH} 7.4$ using $\mathrm{NaOH}$ with cyanide $(2 \mathrm{mM})$ and iodoacetic acid $(1 \mathrm{mM})$. The Ml solution contained iodoaectic acid $(1 \mathrm{mM})$ to inhibit glyceraldehyde 3-phosphate dehydrogenase and cyanide $(2 \mathrm{mM})$ to block electron transfer from complex IV of the electron transport chain. The Ml solution also lacked the metabolic substrates, glucose and pyruvate, which are necessary for ATP generation. ${ }^{11}$ Isolated myocytes were superfused with MI solution for $7 \mathrm{~min}$, followed by $10 \mathrm{~min}$ of re-energisation by superfusion with Tyrode solution to simulate reperfusion injury. For assays in which MI was used, all cardiac myocytes were subjected to the $\mathrm{Ml} /$ reperfusion protocol, and the control group were populations of cardiac myocytes that had not had prior hypothermia or drug treatment.

Temperature preconditioning of isolated ventricular myocytes. For the purposes of temperature preconditioning, isolated ventricular myocytes in Tyrode solution (approximately 0.4 million cells $/ \mathrm{ml}$ ) was placed in a water bath to reach a final temperature of $16^{\circ} \mathrm{C}$ for a 2-min period. The myocytes were then immersed in a water bath at $37^{\circ} \mathrm{C}$ for $3 \mathrm{~min}$. Temperature preconditioning using confocal microscopy of the mt-cpYFP superoxide biosensor was conducted within the microscope chamber and was achieved by superfusing myocytes with Tyrode solution cooled to $16^{\circ} \mathrm{C}$ for $2 \mathrm{~min}$ and $37^{\circ} \mathrm{C}$ for $3 \mathrm{~min}$ for two cycles.

Measurement of myocyte contractile recovery. Isolated ventricular myocytes were placed in a diamond-shaped chamber with a parallel alignment of two platinum electrodes for electric-field stimulation at $1 \mathrm{~Hz}$ (physiological stimulator, Farnell, Leeds, UK) on the stage of a Nikon (Kingston upon Thames, UK) inverted light microscope. Myocytes were perfused at $33-35^{\circ} \mathrm{C}$ at a rate of $4.5 \mathrm{ml} / \mathrm{min}$. Viable rod-shaped cells that were synchronously contracting in response to electric-field stimulation were identified, and the contractile response of these myocytes was then tracked throughout 7 min of superfusion with $\mathrm{Ml}$ and $10 \mathrm{~min}$ of re-energisation with Tyrode solution. Those myocytes that were contractile at $10 \mathrm{~min}$ of re-energisation were considered to have recovered their contractile function. Myocytes that did not respond to the electrical field stimulation or contracted asynchronously were deemed not to have recovered contractile function. For the purposes of temperature preconditioning, myocytes were temperature preconditioned before being placed in the chamber. For DNP preconditioning, myocytes were superfused within the chamber for $5 \mathrm{~min}$ in substrate-free Tyrode containing DNP $(50 \mu \mathrm{M})$, followed by $5 \mathrm{~min}$ in substrate-free Tyrode solution immediately before MI. Where CsA was used, $4 \mu \mathrm{M}$ was present throughout the entire experiment.

Fluorescence imaging. Isolated ventricular myocytes were placed in the chamber of a Nikon (Eclipse TE200) inverted epifluorescence microscope. Myocytes were superfused with Tyrode solution at a rate of $4.5 \mathrm{ml} / \mathrm{min}$ at $34^{\circ} \mathrm{C}$ (Dagan, Minneapolis, MN, USA). For calcium imaging experiments, myocytes were stimulated in an electrical field at $1 \mathrm{~Hz}$ (Research Stimulator, Harvard Apparatus, Kent, UK). For fluorescence imaging, myocytes were illuminated at the appropriate wavelength using a monochromator (PTI deltaRAM), emission was measured using a CCD camera (Photometrics Cascade: 512B; Roper Scientific, Inc., Tucson, AZ, USA) and EasyRatioPro software was used for all analysis (PTI, Birmingham, NJ, USA).

For calcium imaging, isolated ventricular myocytes were loaded for $20 \mathrm{~min}$ at room temperature (approximately $21^{\circ} \mathrm{C}$ ) in Tyrode solution with the ratiometric calcium-sensitive dye, fura-2AM (Invitrogen, Paisley, UK; $2.5 \mu \mathrm{M}$ in Tyrode solution). Myocytes were imaged with a $\times 20$ fluorescence objective and were alternately excited at 340 and $380 \mathrm{~nm}$. Emission was recorded at $>520 \mathrm{~nm}$. All rodshaped myocytes that responded to the electrical field stimulation $(1 \mathrm{~Hz})$ at the start of the experiment were included in the study. A calcium calibration was achieved by exposing fura-2-loaded and ionomycin $(10 \mu \mathrm{M})$-treated myocytes to a high calcium solution $(10 \mathrm{mM})$, followed by exposure to a calcium-free solution (EGTA, $15 \mathrm{mM})$. Fluorescence was measured after $10 \mathrm{~min}$ of re-energisation, when the stimulator had been turned off to measure basal cellular calcium levels. A calcium calibration 
kit was used to estimate free calcium (Invitrogen). This method of calcium calibration is crude and was only intended to give a rough estimate of cellular calcium concentrations for the purposes of comparison.

For measurements of mPTP opening, a well-established assay was used, which takes advantage of both the facts that TMRM, continuously illuminated at $546 \mathrm{~nm}$, will produce ROS that induce MPTP opening, and that TMRM fluorescence reports changes in mitochondrial membrane potential. ${ }^{8}$ Isolated ventricular myocytes were loaded with the fluorescent dye TMRM $(5 \mu \mathrm{M})$ at $37^{\circ} \mathrm{C}$ for $15 \mathrm{~min}$, washed and imaged using a $\times 60$ oil immersion objective. TMRM-loaded myocytes were continuously excited at $546 \mathrm{~nm}$ and the emission measured using a long-pass filter at $>560 \mathrm{~nm}$. Depolarisation of the mitochondrial membrane potential was reported by an increase in TMRM fluorescence, as it dequenched on leaving the mitochondria.

For experiments with relatively low TMRM $(50 \mathrm{nM})$, a low sampling rate $(0.2 \mathrm{~Hz})$ was used to decrease TMRM excitation. Xanthine $(2 \mathrm{mM})$ and xanthine oxidase $(20 \mathrm{mU})$ were superfused onto the myocytes to provide ROS for induction of MPTP opening. Depolarisation of the mitochondrial membrane potential was reported by a decrease in TMRM fluorescence, as the dye diffused from the mitochondria and the cell.

For the detection of MPTP flickering, isolated ventricular myocytes were loaded with calcein-AM ( $1 \mu \mathrm{M}$; Invitrogen) and cobalt chloride ( $1 \mathrm{mM}$; Invitrogen) for $15 \mathrm{~min}$ at room temperature (approximately $21^{\circ} \mathrm{C}$ ), imaged using a $\times 60$ oil immersion objective and were excited at $488 \mathrm{~nm}$, and the calcein fluorescence emission collected using a long-pass filter $>505 \mathrm{~nm}$. Three images of calcein fluorescence were measured at times 0,10 and $30 \mathrm{~min}$ from rod-shaped myocytes. When mPTP flickering occurs, calcein is redistributed to the cytoplasm and quenched by cobalt, which is measured as a decrease in fluorescence.

For measuring mitochondrial ROS formation, ventricular myocytes were infected with adenovirus encoding mt-cpYFP for $48 \mathrm{~h}$. Ventricular myocytes were cultured in media 199 supplemented with ITS $+3(2 \mathrm{mg} / \mathrm{ml})$, carnitine $(2 \mathrm{mM})$, creatine $(5 \mathrm{mM})$, taurine $(5 \mathrm{mM}), \mathrm{T} 3(1.5 \mathrm{pM})$, and penicillin and streptomycin ( $100 \mathrm{units})$ at $37^{\circ} \mathrm{C}$ and $5 \% \mathrm{CO}_{2}$. After $48 \mathrm{~h}$, approximately $70 \%$ of myocytes were infected. The mt-cpYFP fluorescence was measured using confocal microscopy on an Olympus IX70 confocal microscope (Olympus, Southend-on-Sea, UK) with a $\times 60$ oil immersion objective, and myocytes were excited using the argon laser (Melles Griot, Albuquerque, NW, USA) at $488 \mathrm{~nm}$, and the mt-cpYFP emission was collected at $0.2 \mathrm{~Hz}$ using a long-pass filter $>515 \mathrm{~nm}$.

For co-localisation analysis, $48 \mathrm{~h}$ infected myocytes were loaded with MitoTracker deep red $(500 \mathrm{nM}$; Invitrogen) for $20 \mathrm{~min}$ at room temperature (approximately $21^{\circ} \mathrm{C}$ ) and washed. For measurement of the MitoTracker deep red fluorescence, myocytes were illuminated using red helium-neon laser (Melles Griot) at $633 \mathrm{~nm}$, and fluorescence was measured using a $660 \mathrm{~nm}$ long-pass filter.

Western blotting. A sample of cardiac myocytes were temperature preconditioned in the presence or absence of ERK $1 / 2$ inhibitors, either PD98095 $(10 \mu \mathrm{M})$ or U0126 $(0.5 \mu \mathrm{M})$, or the ROS scavenger MGP $(300 \mu \mathrm{M})$. The myocytes were then lysed, and the collected protein was subjected to SDSPAGE. Equal amounts of protein, measured by the Bradford assay, from each treatment group were loaded in each well. The phosphorylation state of ERK $1 / 2$ (p42/p44) protein level was determined for each treatment group. Both ERK1/2 antibodies were obtained from Cell Signaling Technology (Hitchin, UK) and were used in accordance with the manufacturer's instructions. The pERK levels were normalised to the respective total-ERK levels in each well. Relative densitometry was determined using Felix, a computerised software analysis package.

Statistics. Values are expressed as mean \pm S.E.M. Data were analysed by an unpaired Student's t-test or a one-way analysis of variance, as appropriate, $P<0.05$ was considered significant. Where data is expressed as 'normalised to control', this indicates that all data values were divided by the mean control value for the data set.

Acknowledgements. We are grateful to the British Heart Foundation for research funding for this project (FS/08/045/25508), Dr. Heping Cheng for the gift of mt-cpYFP, and Mrs. Sonja Khemiri for assistance with adenovirus preparation.

1. Halestrap AP. What is the mitochondrial permeability transition pore? J Mol Cell Cardiol 2009; 46: 821-831.

2. Halestrap AP, Clarke SJ, Javadov SA. Mitochondrial permeability transition pore opening during myocardial reperfusion-a target for cardioprotection. Cardiovasc Res 2004; 61: 372-385.
3. Campanella M, Casswell E, Chong S, Farah Z, Wieckowski MR, Abramov AY et al. Regulation of mitochondrial structure and function by the F1Fo-ATPase inhibitor protein, IF1. Cell Metab 2008; 8: 13-25.

4. Baines $C P$. The mitochondrial permeability transition pore and ischemia-reperfusion injury. Basic Res Cardiol 2009; 104: 181-188

5. Halestrap AP, Clarke SJ, Khaliulin I. The role of mitochondria in protection of the heart by preconditioning. Biochim Biophys Acta 2007; 1767: 1007-1031.

6. Griffiths EJ, Halestrap AP. Protection by cyclosporin A of ischemia/reperfusion-induced damage in isolated rat hearts. $J$ Mol Cell Cardiol 1993; 25: 1461-1469.

7. Piot $\mathrm{C}$, Croisille $\mathrm{P}, \mathrm{Staat} P$, Thibault $\mathrm{H}$, Rioufol $\mathrm{G}$, Mewton $\mathrm{N}$ et al. Effect of cyclosporine on reperfusion injury in acute myocardial infarction. N Engl J Med 2008; 359: 473-481.

8. Hausenloy DJ, Yellon DM, Mani-Babu S, Duchen MR. Preconditioning protects by inhibiting the mitochondrial permeability transition. Am J Physiol Heart Circ Physiol 2004; 287: $\mathrm{H} 841-\mathrm{H} 849$.

9. Petronilli V, Miotto G, Canton M, Brini M, Colonna R, Bernardi P et al. Transient and long-lasting openings of the mitochondrial permeability transition pore can be monitored directly in intact cells by changes in mitochondrial calcein fluorescence. Biophys $J 1999$; 76: $725-734$

10. Huser J, Blatter LA. Fluctuations in mitochondrial membrane potential caused by repetitive gating of the permeability transition pore. Biochem J 1999; 343: 311-317.

11. Rodrigo GC, Lawrence CL, Standen NB. Dinitrophenol pretreatment of rat ventricular myocytes protects against damage by metabolic inhibition and reperfusion. $J \mathrm{Mol}$ Cell Cardiol 2002; 34: 555-569.

12. Ji L, Fu F, Zhang L, Liu W, Cai $X$, Zhang $L$ et al. Insulin attenuates myocardial ischemia/ reperfusion injury via reducing oxidative/nitrative stress. Am J Physiol Endocrinol Metab 2010; 298: E871-E880.

13. Murry $C E$, Jennings RB, Reimer KA. Preconditioning with ischemia: a delay of lethal cell injury in ischemic myocardium. Circulation 1986; 74: 1124-1136.

14. Khaliulin I, Clarke SJ, Lin H, Parker J, Suleiman MS, Halestrap AP. Temperature preconditioning of isolated rat hearts-a potent cardioprotective mechanism involving a reduction in oxidative stress and inhibition of the mitochondrial permeability transition pore. J Physiol 2007; 581: 1147-1161.

15. Hale SL, Kloner RA. Myocardial temperature reduction attenuates necrosis after prolonged ischemia in rabbits. Cardiovasc Res 1998; 40: 502-507.

16. Hale SL, Kloner RA. Mild hypothermia as a cardioprotective approach for acute myocardial infarction: laboratory to clinical application. J Cardiovasc Pharmacol Ther 2011; 16: 131-139.

17. Abendschein DR, Tacker WA Jr, Babbs CF. Protection of ischemic myocardium by wholebody hypothermia after coronary artery occlusion in dogs. Am Heart J 1978; 96: 772-780.

18. Gotberg M, Olivecrona GK, Engblom H, Ugander M, van der Pals J, Heiberg E et al. Rapid short-duration hypothermia with cold saline and endovascular cooling before reperfusion reduces microvascular obstruction and myocardial infarct size. BMC Cardiovasc Disord 2008; 8: 7

19. Polderman KH. Mechanisms of action, physiological effects, and complications of hypothermia. Crit Care Med 2009; 37: S186-202.

20. Jones RN, Reimer KA, Hill ML, Jennings RB. Effect of hypothermia on changes in highenergy phosphate production and utilization in total ischemia. J Mol Cell Cardiol 1982; 14: 123-130.

21. Hausenloy DJ, Lim SY, Ong SG, Davidson SM, Yellon DM. Mitochondrial cyclophilin-D as a critical mediator of ischaemic preconditioning. Cardiovasc Res 2010; 88: 67-74.

22. Hausenloy DJ, Tsang A, Mocanu MM, Yellon DM. Ischemic preconditioning protects by activating prosurvival kinases at reperfusion. Am J Physiol Heart Circ Physiol 2005; 288 H971-H976.

23. Solenkova NV, Solodushko V, Cohen MV, Downey JM. Endogenous adenosine protects preconditioned heart during early minutes of reperfusion by activating Akt. Am J Physiol Heart Circ Physiol 2006; 290: H441-H449.

24. Yang X, Liu Y, Yang XM, Hu F, Cui L, Swingle MR et al. Cardioprotection by mild hypothermia during ischemia involves preservation of ERK activity. Basic Res Cardiol 2011; 106: 421-430.

25. Wang W, Fang $\mathrm{H}$, Groom L, Cheng A, Zhang W, Liu J et al. Superoxide flashes in single mitochondria Cell 2008; 134: 279-290.

26. M Crompton. Bax, Bid and the permeabilization of the mitochondrial outer membrane in apoptosis. Curr Opin Cell Biol 2000; 12: 414-419.

27. Cour M, Loufouat J, Paillard M, Augeul L, Goudable J, Ovize M et al. Inhibition of mitochondrial permeability transition to prevent the post-cardiac arrest syndrome: a preclinical study. Eur Heart J 2011; 32: 226-235.

28. Hausenloy D, Boston-Griffiths E, Yellon D. Cyclosporin A and cardioprotection: from investigative tool to therapeutic agent. Br J Pharmacol 2011; 165: 1235-1245.

29. Huser J, Blatter LA. Fluctuations in mitochondrial membrane potential caused by repetitive gating of the permeability transition pore. Biochem J 1999; 343: 311-317.

30. Hausenloy D, Wynne A, Duchen M, Yellon D. Transient mitochondrial permeability transition pore opening mediates preconditioning-induced protection. Circulation 2004; 109: 1714-1717.

31. Hausenloy DJ, Tsang A, Yellon DM. The reperfusion injury salvage kinase pathway: common target for both ischemic preconditioning and postconditioning. Trends Cardiovasc Med 2005; 15: 69-75.

32. Kelly FE, Nolan JP. The effects of mild induced hypothermia on the myocardium: systematic review. Anaesthesia 2010; 65: 505-515. 
33. Khaliulin I, Halestrap AP, Suleiman MS. Temperature preconditioning is optimal at 26 degrees $\mathrm{C}$ and confers additional protection to hypothermic cardioplegic ischemic arrest. Exp Biol Med (Maywood) 2011; 236: 736-745.

34. Piper HM, Abdallah Y, Schafer C. The first minutes of reperfusion: a window of opportunity for cardioprotection. Cardiovasc Res 2004; 61: 365-371.

35. Altschuld RA, Wenger WC, Lamka KG, Kindig OR, Capen CC, Mizuhira V et al. Structura and functional properties of adult rat heart myocytes lysed with digitonin. J Biol Chem 1985; 260: 14325-14334.

36. Dong Z, Saikumar P, Weinberg JM, Venkatachalam MA. Calcium in cell injury and death Annu Rev Pathol 2006; 1: 405-434.

37. Griffiths EJ, Halestrap AP. Mitochondrial non-specific pores remain closed during cardiac ischaemia, but open upon reperfusion. Biochem J 1995; 307: 93-98.

38. Al-Nasser I, Crompton M. The entrapment of the $\mathrm{Ca} 2+$ indicator arsenazo III in the matrix space of rat liver mitochondria by permeabilization and resealing. $\mathrm{Na}+$-dependen and -independent effluxes of $\mathrm{Ca} 2+$ in arsenazo III-loaded mitochondria. Biochem J 1986; 239: $31-40$.
39. Halestrap AP. A pore way to die: the role of mitochondria in reperfusion injury and cardioprotection. Biochem Soc Trans 2010; 38: 841-860.

40. Ali SS, Marcondes MC, Bajova H, Dugan LL, Conti B. Metabolic depression and increased reactive oxygen species production by isolated mitochondria at moderately lower temperatures. J Biol Chem 2010; 285: 32522-32528.

41. Riess ML, Camara AK, Kevin LG, An J, Stowe DF. Reduced reactive $\mathrm{O} 2$ species formation and preserved mitochondrial $\mathrm{NADH}$ and $[\mathrm{Ca} 2+]$ levels during short-term 17 degrees $\mathrm{C}$ ischemia in intact hearts. Cardiovasc Res 2004; 61: 580-590.

Cell Death and Disease is an open-access journal published by Nature Publishing Group. This work is licensed under the Creative Commons Attribution-NonCommercial-No Derivative Works 3.0 Unported License. To view a copy of this license, visit http://creativecommons.org/licenses/by-nc-nd/3.0/ 